\title{
Design of Compact Dual-Polarized Antennas for MIMO Handsets
}

\author{
Wenhua Chen, Xin Wang, and Zhenghe Feng \\ State Key Laboratory on Microwave and Digital Communications, Tsinghua University, Beijing 100084, China \\ Correspondence should be addressed to Wenhua Chen, chenwh@tsinghua.edu.cn
}

Received 11 June 2011; Accepted 19 July 2011

Academic Editor: Yuan Yao

Copyright (C) 2012 Wenhua Chen et al. This is an open access article distributed under the Creative Commons Attribution License, which permits unrestricted use, distribution, and reproduction in any medium, provided the original work is properly cited.

\begin{abstract}
A design method of compact dual-polarized antennas has been proposed for multiple input and multiple output (MIMO) handset application. For the sake of high isolation in dual polarizations, a printed monopole and a hybrid slot antenna are combined using a coplanar waveguide $(\mathrm{CPW})$ and microstrip hybrid feeding structure. The proposed topology will result in orthogonal current distribution modes for the different polarizations, which effectively reduces the mutual coupling of the two modes. The operation mechanism of the feeding structure is analyzed in principle and verified by simulation. Simulated and measured results show this compact dual-polarized antenna achieves isolation between the two ports of better than $25 \mathrm{~dB}$, and the relative bandwidth is wider than $23.5 \%$.
\end{abstract}

\section{Introduction}

Researches on multiple input and multiple output (MIMO) technology are very popular in wireless communication area, since more than 1 Gbps data transmission rate can be achieved using MIMO technology. As revealed in many literatures $[1,2]$, the performance of MIMO system sensitively depends on the status of the channel, including radio propagation, multipath effect, and antenna radiation pattern. Recently much attention has been attracted to antenna selection in MIMO system because of its better performance and low cost [3]. Compared with the other components in wireless transceiver, the antennas are much cheaper. Thereby, we can use adequate antennas to perform better channel status and approach the maximum capacity of MIMO system. Several antenna selection schemes [4-8] have been studied extensively so far, such as space selection, radiation pattern selection and polarization selection. In these selection schemes, the polarization selection has more potential for future application due to its compact size and easy fabrication. As shown in previous literature $[4,5]$, the capacity of MIMO system can be enhanced by using switchable polarization schemes in the redundant elements of MIMO system.

In this paper, based on our proposed dual-polarized antenna in [9], a detailed design method of compact dualpolarized MIMO antennas is presented and discussed. This antenna combines a printed monopole $[10,11]$ fed by coplanar waveguide and a hybrid slot antenna [12-15] fed by coupling microstrip line to generate vertical and horizontal polarized radiation, respectively. Low mutual coupling is guaranteed by the orthogonality of different current modes. The structure is planar and can be easily fabricated by general printed circuit board (PCB) techniques, thus it is convenient to be integrated into portable transceivers. Parametric studies have been carried out to investigate the dimensional sensitivities of the proposed antenna structure. Measured $S$-parameters and radiation patterns are presented to validate the proposed design.

\section{Antenna Configuration and Mechanism}

As we know, printed monopole antennas have been widely used in handsets. Meanwhile, quarter wavelength slot antennas with microstrip feeding line are easy to be integrated into PCB, so they are also popular for volume limited and wideband application. If these two types of antennas can be combined together, a compact dual polarized antenna with inherited low design complexity and fabrication cost can be realized for mobile terminals. As shown in Figure 1, the current distribution of printed monopole antennas and quarter wavelength slot antennas are presented and compared schematically. For a symmetrical feeding structure, the current distribution in monopole antenna is symmetrical 


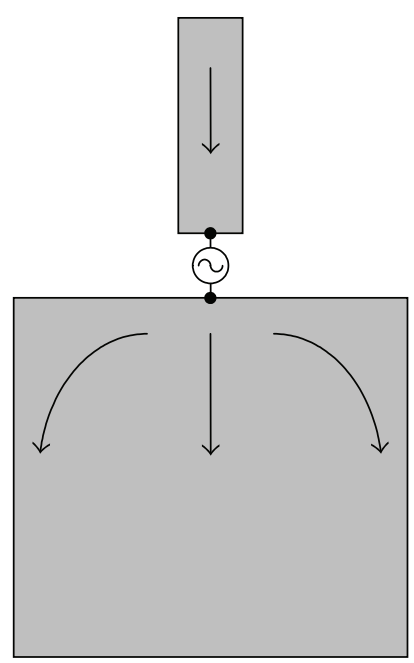

(a)

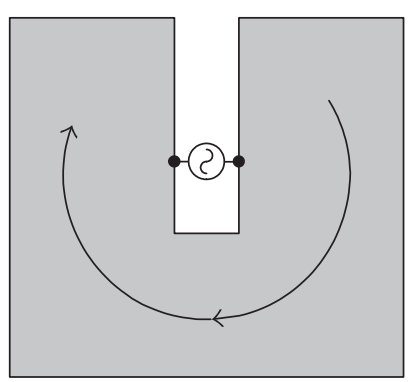

(b)
Figure 1: The current distribution of printed monopole and quarter wavelength slot antenna.

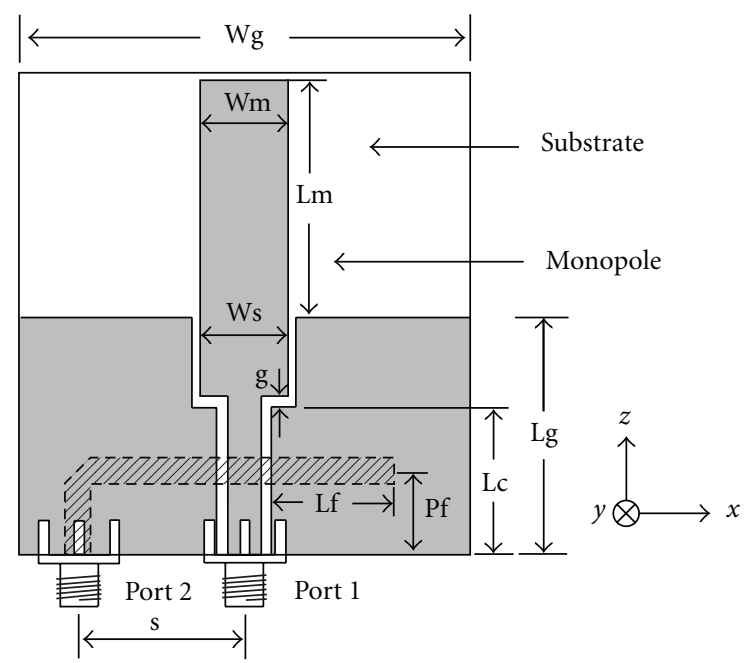

FIGURE 2: Sketch map of proposed antenna.

and in phase. With respect to slot antenna, the current distribution remains symmetrical but out of phase. Supposing these two antennas can be combined together, the isolation between the two antennas can be guaranteed. Unfortunately, there is a dilemma for implementing the required current distributions for both antennas simultaneously.

In order to achieve a compact dual-polarized antenna with high isolation and illuminate the dilemma in feeding the structure, a novel structure is proposed in this paper. The proposed antenna is designed to operate at $2.35 \mathrm{GHz}$ and the detailed configuration is shown in Figure 2, which exhibits the needed current distribution and still has a symmetrical feeding structure approximately. The antenna is printed on a substrate with relative permittivity 2.65 and thickness of $1 \mathrm{~mm}$, the detailed antenna dimensions are listed in Table 1. As shown in Figure 2, on the front side of the substrate

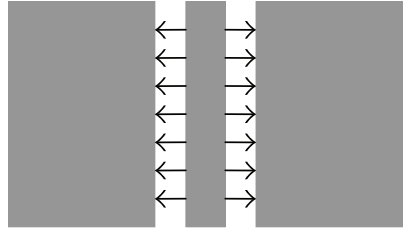

(a)

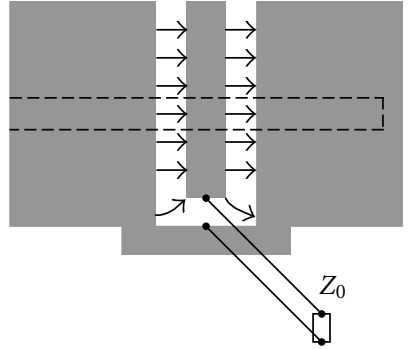

(b)
FIGURE 3: Electromagnetic field distribution, (a) CPW feeding port and (b) microstrip feeding port.

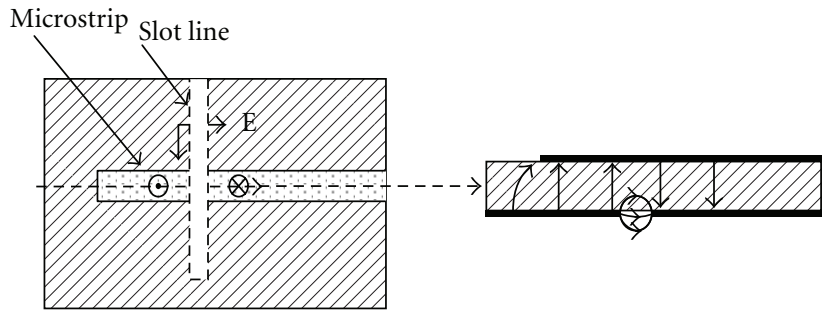

(a)

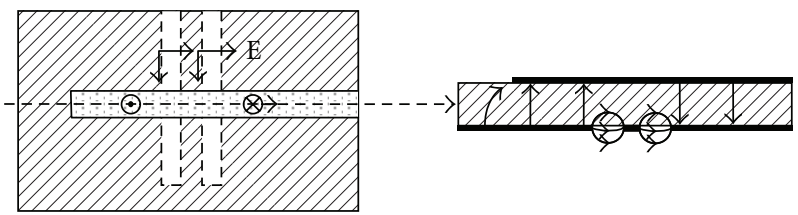

(b)

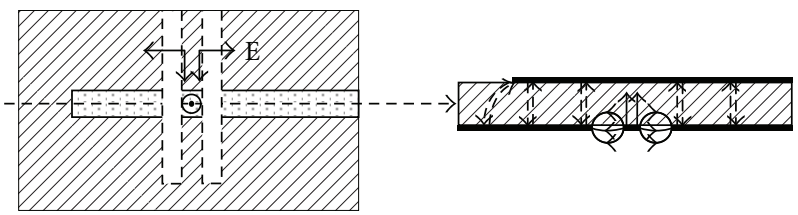

(c)

FIGURE 4: Slot line to microstrip transition, (a) single slot line, (b) paralleled slot lines with equivalent and in-phase fields, and (c) paralleled slot lines with equivalent and out-of-phase fields.

there is a monopole fed by a $50 \mathrm{Ohm}$ CPW. The lengths of the monopole $(\mathrm{Lm})$ and the ground $(\mathrm{Lg})$ are chosen at about one quarter wavelength. By welding an SMA connecter to the CPW, the two halves of the ground will be shorted at the bottom and form a hybrid-slot which is also about one quarter wavelength long and actually united by two paralleled slots as illustrated in Figure 2. To excite this slot antenna, a $50 \mathrm{Ohm}$ coupling microstrip line is printed on the back side of the substrate.

To obtain the required current distributions for the dual polarizations, a coplanar waveguide (CPW) topology is used for the printed monopole antenna. In addition, this CPW topology can be regarded as a portion of the slot antennas. In the initial design stage, the lengths of monopole and ground plane both equal to quarter wavelength. An SMA (SubMiniature version A) connector is utilized for feeding 


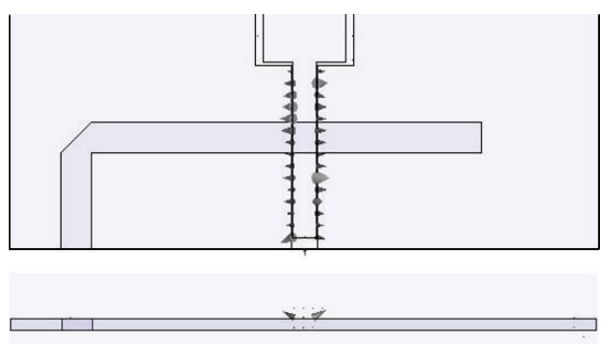

(a)

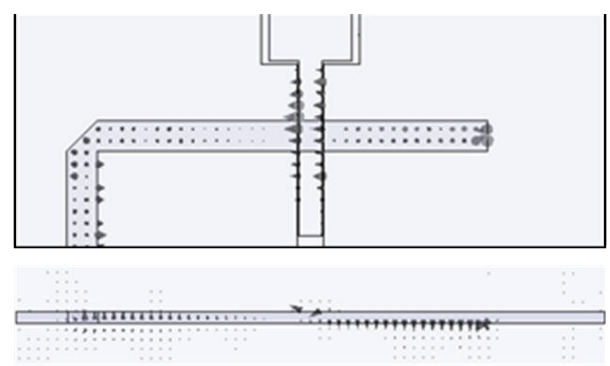

(b)

FIGURE 5: Electric field distribution with different feeding ports: (a) CPW and (b) microstrip.

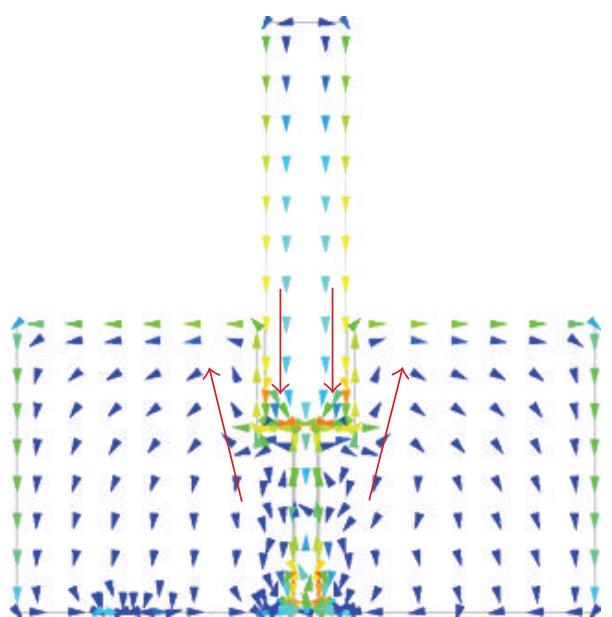

(a)

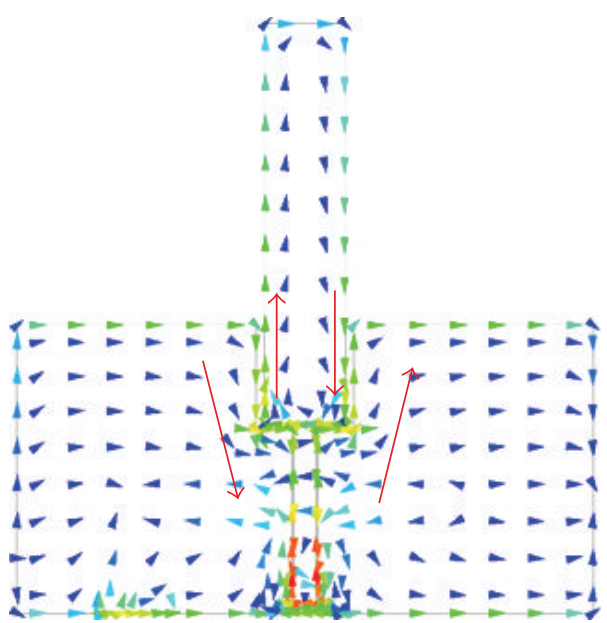

(b)

FIgURE 6: Current distribution in different modes, (a) CPW feeding and (b) microstrip feeding.

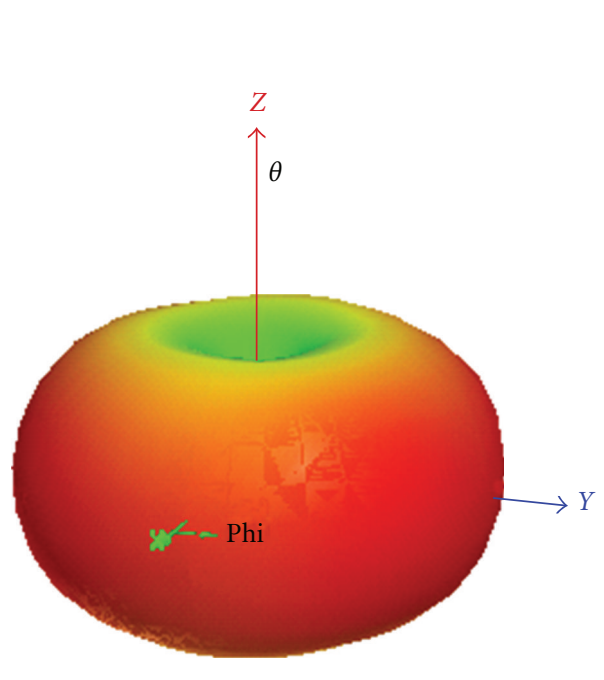

(a)

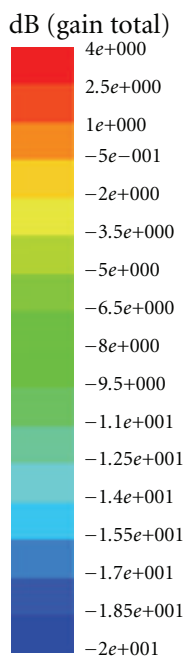

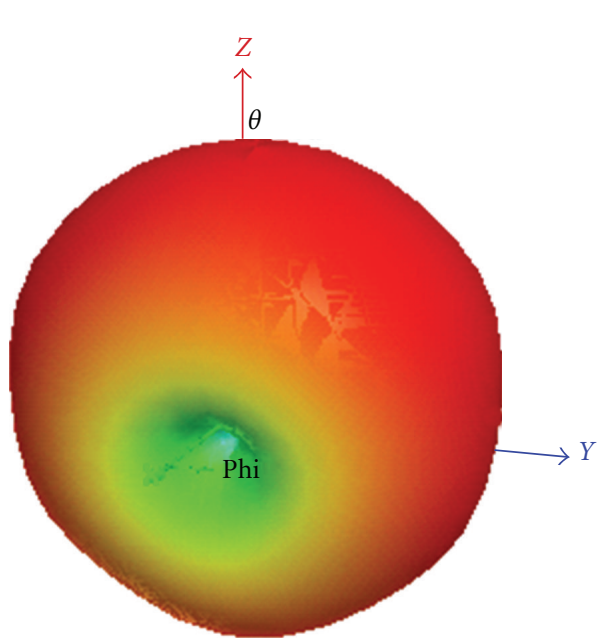

(b)

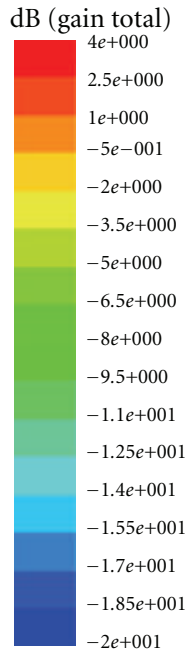

$-2 e+001$

FIGURE 7: Simulated 3D radiation patterns fed by different ports, (a) CPW and (b) microstrip. 
TABLe 1: Antenna dimensions.

\begin{tabular}{lccccc}
\hline Dimension & Size $(\mathrm{mm})$ & Dimension & Size $(\mathrm{mm})$ & Dimension & Size $(\mathrm{mm})$ \\
\hline Wg & 50 & Lg & 25 & $\mathrm{~d}$ & gm \\
Wm & 7 & Lm & 25 & g & 0.7 \\
W & 2 & Lc & 15.5 & Pf & 0.1 \\
Wf & 2.6 & Lf & 15 & 9.5 \\
\hline
\end{tabular}

port, in which two-sided ground planes are connected together via its outer conductor. In Figure 3, the electric field distribution within CPW feeding mode and microstrip feeding mode are plot in theory. As illustrated in Figure 3, in the CPW feeding, the electric fields are symmetrical and in the opposite direction whereas the electric fields using microstrip feeding are in the same direction. Thereby, the current distribution induced by the combination of CPW and microstrip feeding is consistent with the requirements of dual polarized antennas. Actually the derived slot antenna consists of two neighboring slot antennas. Since the spacing between the two slots is very small, the magnetic current excited by the microstrip line will be equivalent in terms of magnitude and phase. Finally, the two slots can be viewed as a single hybrid slot for far field radiation.

The transition from slot line to microstrip is presented in Figure 4(a), and the electric field distributions in slot line as well as the microstrip line which is coupled from the slot line are shown. Furthermore, as shown in Figure 4(b), considering two paralleled and close slot lines, in which the electric fields are equal and in phase, as a result, the electric fields induced by the same feeding slot line will have the same characteristics. Consequently the combined feeding effect of two slot lines will be equivalent to the single slot line. Oppositely, in Figure 4(c), if the electric fields are equal and out of phase, the coupled electric field in microstrip line will be canceled with each other, no electronic field will transmit on the microstrip line. Based on this analytical investigation, the microstrip feeding port is shielded from the CPW port, which means that high isolation between the CPW and microstrip feeding ports can be achieved.

\section{Experimental Results}

To validate the above analysis, the proposed structure is simulated in HFSS firstly. Figure 5 shows the electric field distributions in top view and cross-section, which agree well with the above analysis. In the case of CPW feeding, the electric field in the two slots is an even mode. As we expect, the coupling between the two ports is very small and can be neglected. On the other hand, when the microstrip feeding port is active, the current distribution in two slots is an odd mode. Figure 6 shows the simulated current distributions at $2.35 \mathrm{GHz}$ with one port excited and the other is terminated with $50 \mathrm{Ohm}$ matching load. When the monopole is excited, the current flows on the left and right halves are mostly symmetrical and in phase. In this situation, the monopole antenna can radiate efficiently, and a vertical polarized and almost omnidirectional radiation pattern is realized. While with the hybrid slot antenna excited, the

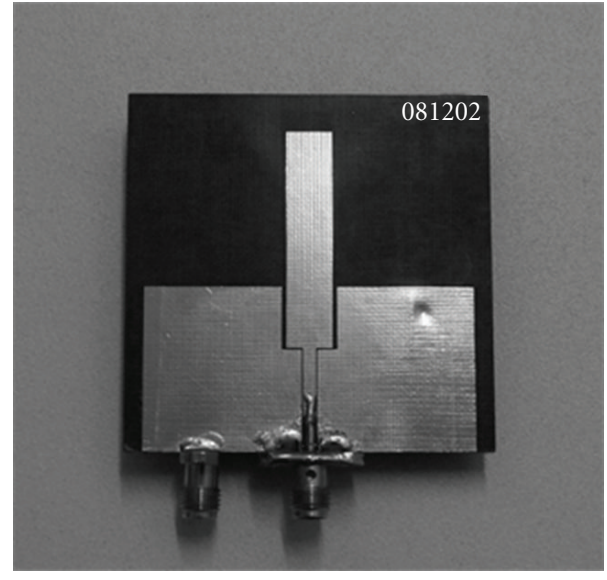

FIGURE 8: The prototype picture.

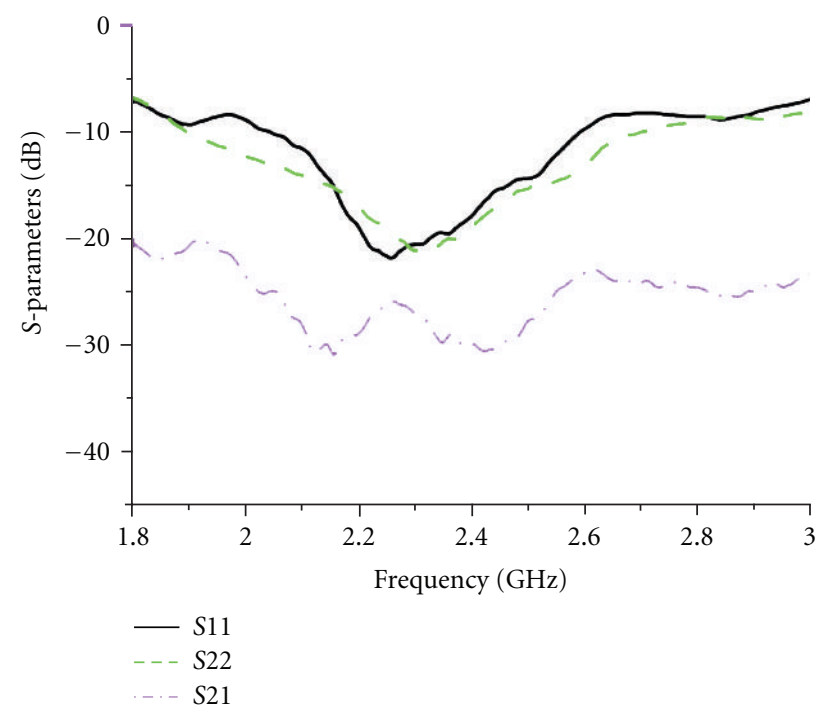

FIGURE 9: Measured $S$-parameters.

current flows on the two edges of the monopole are mostly 180 degrees out of phase, thus the monopole antenna is idle at this time. Consequently, the horizontal polarized radiation is attributed to the current distribution on the ground plane. Herein, electric fields in feeding ports and current distributions on the metal planes both demonstrate the operation mechanism of the proposed feeding topology, which leads to a high isolation between dual polarizations.

Generally wider monopole results in a wider bandwidth; however, the isolation between dual polarizations will be 


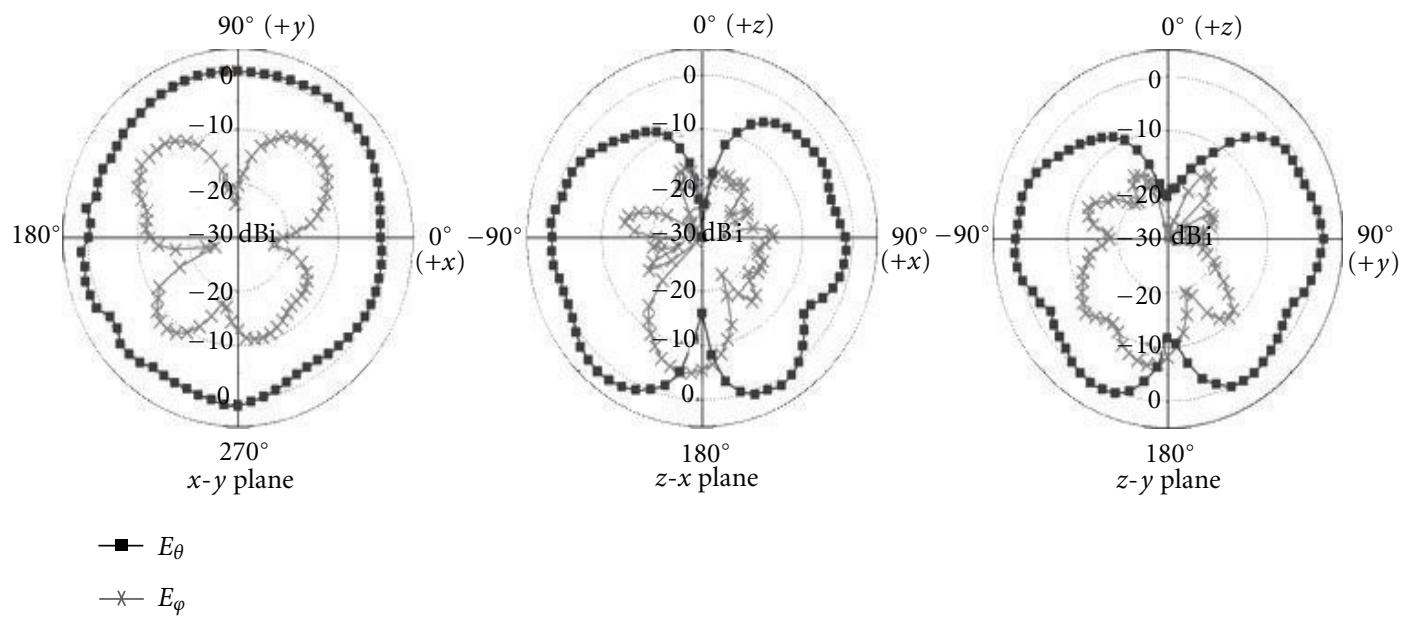

FIGURE 10: Measured radiation patterns of the monopole.
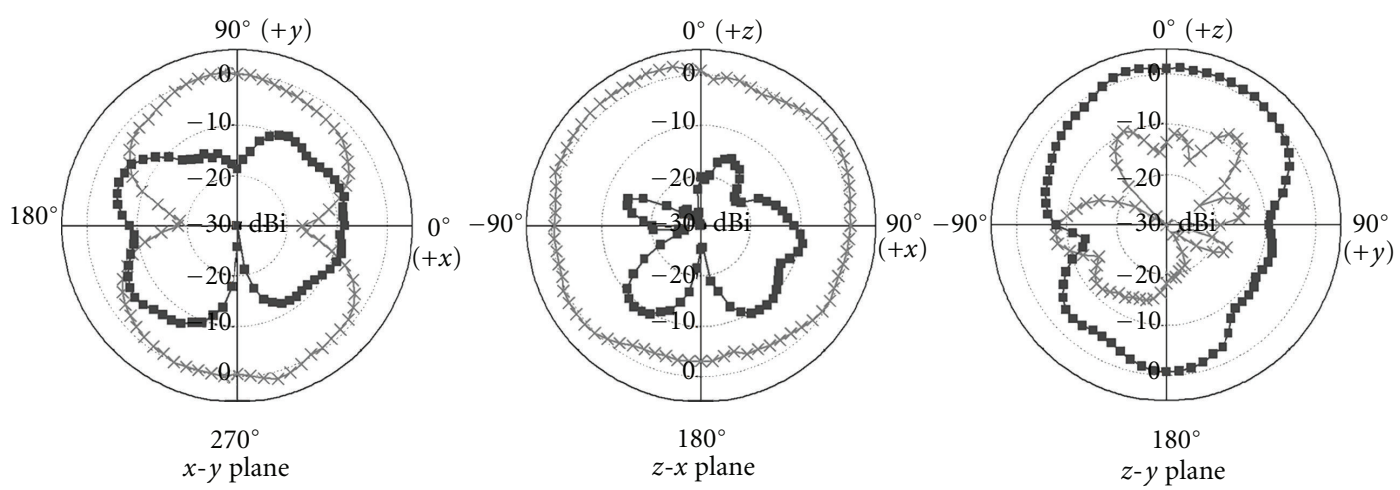

$-E_{\theta}$

$\times E_{\varphi}$

FIGURE 11: Measured radiation patterns of the hybrid slot antenna.

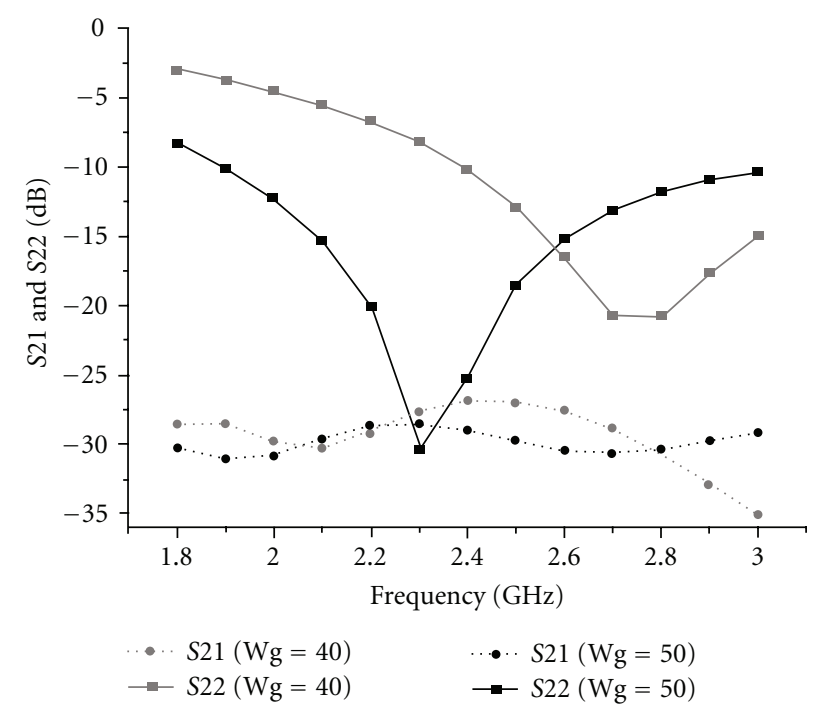

(a)

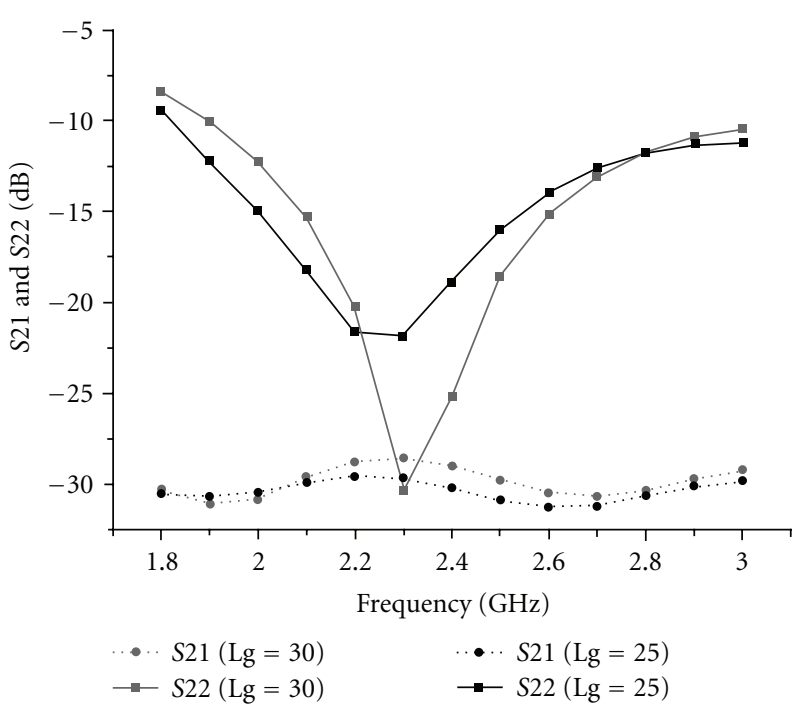

(b)

FIGURE 12: The effect of the ground dimension, (a) ground width Wg and (b) ground length Lg. 


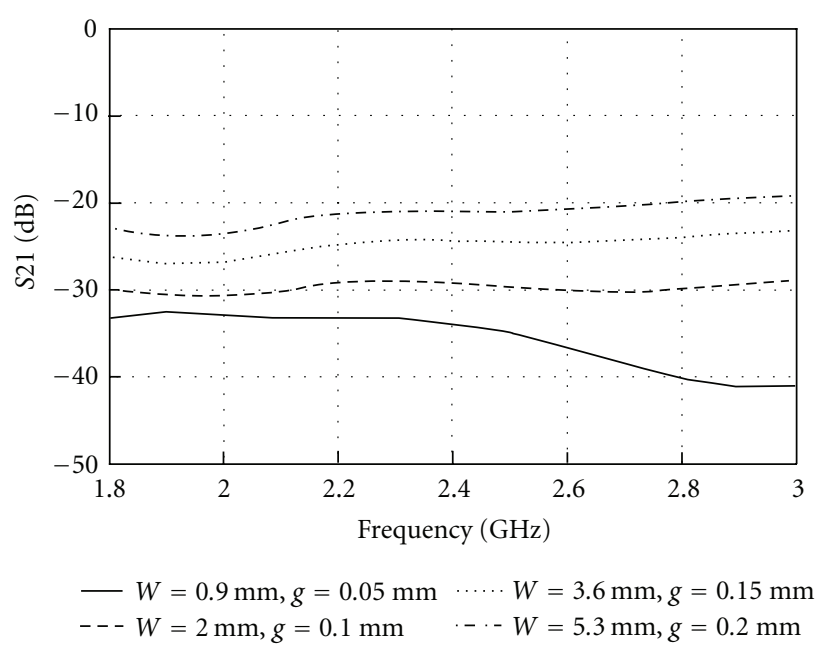

(a)

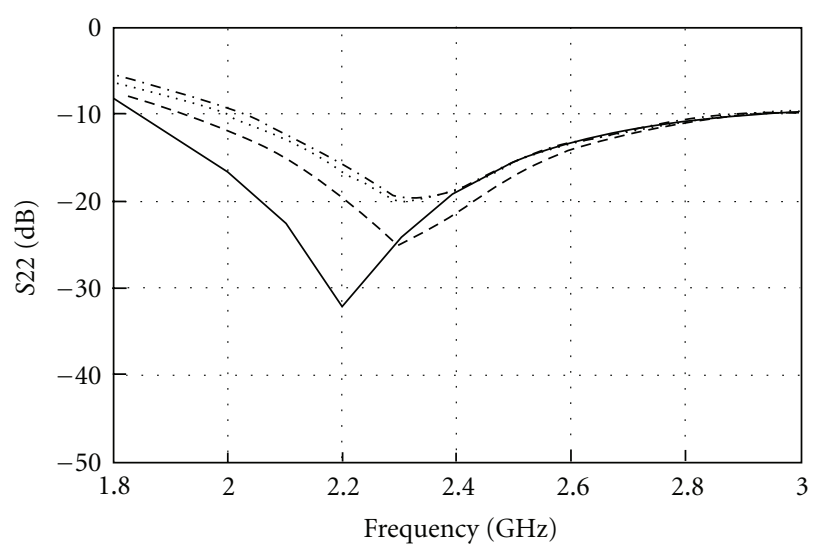

$-W=0.9 \mathrm{~mm}, g=0.05 \mathrm{~mm} \cdots W=3.6 \mathrm{~mm}, g=0.15 \mathrm{~mm}$ $---W=2 \mathrm{~mm}, g=0.1 \mathrm{~mm} \quad \cdots-W=5.3 \mathrm{~mm}, g=0.2 \mathrm{~mm}$

(c)

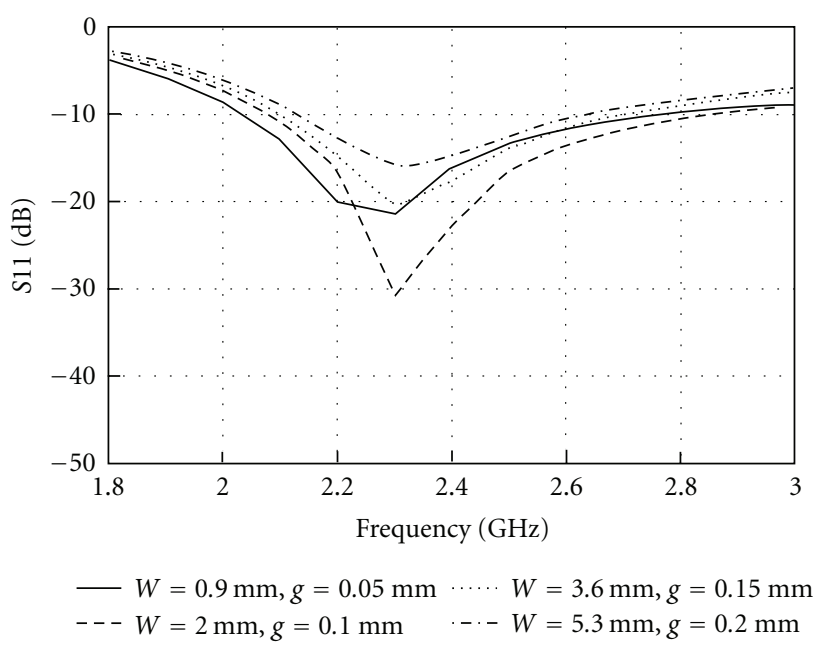

(b)

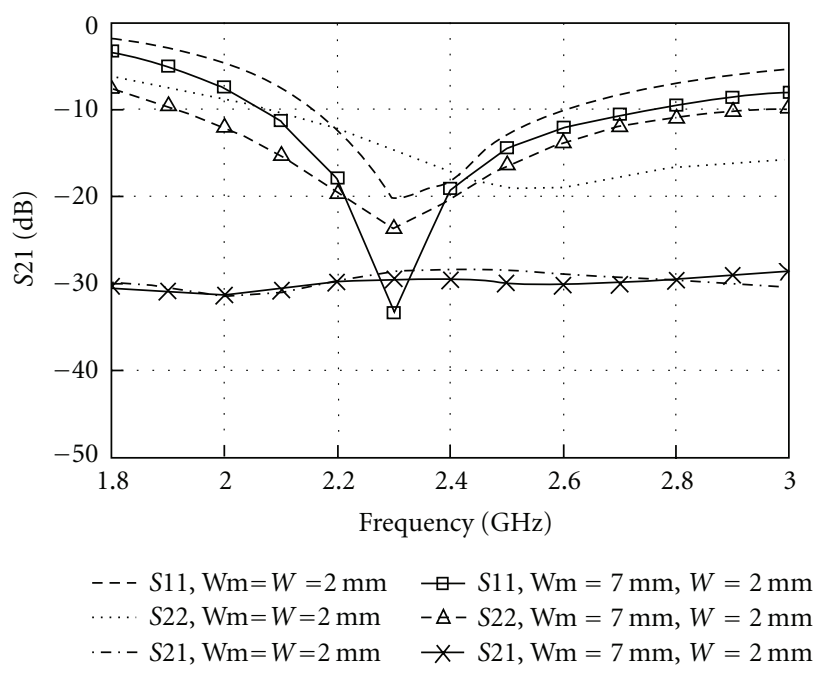

(d)

Figure 13: Parametric study of the dimensions.

deteriorated. As a solution, a ladder configuration monopole is adopted in this design. Since the widths of two segments can be tuned separately, the bandwidth matching and isolation optimization can approach the ideal point without tradeoff. Moreover, this ladder structure will increase the length of the slot for a given ground size, which is also helpful for size reduction. In Figure 7, the simulation 3D radiation patterns fed by different ports are presented, and the gain of the antenna is around $4 \mathrm{dBi}$ in both cases.

A prototype of the proposed antenna as shown in Figure 8 was fabricated and tested, the detailed dimensions can be found in Table 1. Figure 9 shows the measured $S$-parameters. The measured $10 \mathrm{~dB}$ return loss bandwidths are $2.04-2.59 \mathrm{GHz}(23.5 \%)$ for the monopole and 1.89$2.70 \mathrm{GHz}(35 \%)$ for the hybrid slot antenna. The mutual coupling between the two ports is less than $-25 \mathrm{~dB}$ across the common bandwidth. Figures 10 and 11 show the measured radiation patterns of the monopole and the hybrid slot antenna at $2.35 \mathrm{GHz}$. The monopole has an omnidirectional vertically polarized pattern in $x-y$ plane and donut-shaped patterns in the elevation planes. The hybrid slot generates bidirectional and horizontally polarized radiation in $x-y$ plane.

Because open-ended quarter wavelength slot antennas are utilized in this design, radiation in some level will appear in the open-ended direction. It can be anticipated that the radiated field is similar to a horizontal located dipole. Limited by the dimension of the ground plane, the reflection effect of the ground plane will not be significant, thus the radiation pattern $z-y$ plane is close to an omnidirectional pattern. Comparing the radiation patterns in different planes and polarizations, it can be seen that these patterns are complementary in space and polarization dimensions. Benefit from these good characteristics, this proposed antenna can be employed for space and angular diversities in MIMO system. 
TABLE 2: The dimensions of $50 \mathrm{Ohm}$ CPW.

\begin{tabular}{lcc}
\hline Width W $(\mathrm{mm})$ & Gap g $(\mathrm{mm})$ & Gap/wavelength \\
\hline 0.9 & 0.05 & 0.011 \\
2.0 & 0.1 & 0.024 \\
3.6 & 0.15 & 0.043 \\
5.3 & 0.2 & 0.064 \\
\hline
\end{tabular}

\section{Parametric Studies}

For the purpose of optimized performance, parametric studies of the dimensions of the ground plane are carried out. As presented in Figure 12(a), the resonant frequency of port 2 relies on the width of the ground plane, which is consistent with above analysis. According to the comparison in Figure 12(b), the length of the ground plane has effect on S22 parameter, the match performance can be improved by increasing the length of the ground plane.

The isolation between two polarizations will be affected by the dimension of CPW. In principle, the characteristic impedance of CPW depends on the ratio of the gap and the central strip width, which provides much freedom for designing. As listed in Table 2, different kinds of $50 \mathrm{Ohm}$ CPW have been presented, as well as the ratio of the gap spacing and the related wavelength. Base on these feeding structures, the isolation $S 21$ between two ports can be calculated, and the simulated results are given in Figure 13(a). From this figure, increasing the width of CPW, that means the spacing between two gaps is wider, the coupling between two ports is stronger than before; however the isolation is still acceptable. In Figures 13(b) and 13(c), the return losses in two ports are computed with different dimensions, obviously the dimensions of CPW will not affect the matching status significantly.

In order to evaluate the performance of the ladder feeding structure, the simulated $S$-parameters with and without ladder are illustrated. It can be seen that the resonant frequency of the monopole does not change, and isolation between the two polarizations is not varied, but the frequency bandwidth of antenna is enhanced. At the same time, owing to the increase of slot length, the resonant frequency of the hybrid slot will move towards lower frequency.

\section{Conclusion}

In this paper a design method of compact dual polarized antennas has been proposed and implemented for MIMO system. To isolate the dual polarizations, a novel feeding structure is invented. Simulated and measured results showed the two polarizations are highly isolated by combiningz the printed monopole and hybrid slot antenna. The proposed antenna will provide better propagation channel and enhance the capacity of MIMO system.

\section{Acknowledgments}

This paper was supported by the National Basic Research Program of China (2010CB32740) and National High Technology Research and Development Program of China
(863 Program) under Contract 2009AA011503, and also the National Science and Technology Major Project of the Ministry of Science and Technology of China (Grant no. 2009ZX03007-003 and 2012ZX03001009-003).

\section{References}

[1] A. J. Paulraj, D. A. Gore, R. U. Nabar, and H. Bolcskei, "An overview of MIMO communications-a key to gigabit wireless," Proceedings of the IEEE, vol. 92, no. 2, pp. 198-217, 2004.

[2] A. N. González and B. Lindmark, "The effect of antenna orientation and polarization on MIMO capacity," in Proceedings of the IEEE Antennas and Propagation Society International Symposium, pp. 434-437, July 2005.

[3] I. Berenguer, X. Wang, and V. Krishnamurthy, "Adaptive MIMO antenna selection via discrete stochastic optimization," IEEE Transactions on Signal Processing, vol. 53, no. 11, pp. 4315-4329, 2005.

[4] C. B. Dietrich, K. Dietze, J. R. Nealy, and W. L. Stutzman, "Spatial, polarization, and pattern diversity for wireless handheld terminals," IEEE Transactions on Antennas and Propagation, vol. 49, no. 9, pp. 1271-1281, 2001.

[5] B. A. Cetiner, J.-Y. Qian, G. P. Li, and F. De Flaviis, "A reconfigurable spiral antenna for adaptive MIMO systems," Eurasip Journal on Wireless Communications and Networking, vol. 2005, no. 3, pp. 382-389, 2005.

[6] A. Grau, J. Romeu, S. Blanch, L. Jofre, H. Jafarkhani, and F. De Flaviis, "Performance enhancement of the alamouti diversity scheme using polarization-reconfigurable antennas in different fading environments," in Proceedings of the IEEE Antennas and Propagation Society International Symposium, pp. 133-136, July 2006.

[7] H.T. Hui, "Practical dual-helical antenna array for diversity/MIMO receiving antennas on mobile handsets," in Proceedings of the IEE Proceedings of Microwaves, Antennas and Propagation, vol. 152, no. 5, pp. 367-372, October 2005.

[8] B. A. Cetiner, H. Jafarkhani, J.-Y. Qian, H. J. Yoo, A. Grau, and F. De Flaviis, "Multifunctional reconfigurable MEMS integrated antennas for adaptive MIMO systems," IEEEE Communications Magazine, vol. 42, no. 12, pp. 62-70, 2004.

[9] X. Wang, W. Chen, and Z. Feng, "Compact dual-polarized antenna combining printed monopole and halfslot antenna for MIMO applications," in Proceedings of the IEEE International Symposium on Antennas and Propagation, pp. 1-4, June 2009.

[10] M. J. Ammann and M. John, "Optimum design of the printed strip monopole," IEEE Antennas and Propagation Magazine, vol. 47, no. 6, pp. 59-61, 2005.

[11] D. Su, J. J. Qian, H. Yang, and D. Fu, "A novel broadband polarization diversity antenna using a cross-pair of folded dipoles," IEEE Antennas and Wireless Propagation Letters, vol. 4, no. 1, pp. 433-435, 2005.

[12] S. I. Latif, L. Shafai, and S. K. Sharma, "Bandwidth enhancement and size reduction of microstrip slot antennas," IEEE Transactions on Antennas and Propagation, vol. 53, no. 3, pp. 994-1003, 2005.

[13] S. K. Sharma, L. Shafai, and N. Jacob, "Investigation of wideband microstrip slot antenna," IEEE Transactions on Antennas and Propagation, vol. 52, no. 3, pp. 865-872, 2004.

[14] Y. Li, Z. Zhang, W. Chen, Z. Feng, and M. F. Iskander, "A dual-polarization slot antenna using a compact CPW feeding 
structure," IEEE Antennas and Wireless Propagation Letters, vol. 9, pp. 191-194, 2010.

[15] K.-L. Wong, H.-C. Tung, and T.-W. Chiou, "Broadband dualpolarized aperture-coupled patch antennas with modified $\mathrm{H}$ shaped coupling slots," IEEE Transactions on Antennas and Propagation, vol. 50, no. 2, pp. 188-191, 2002. 

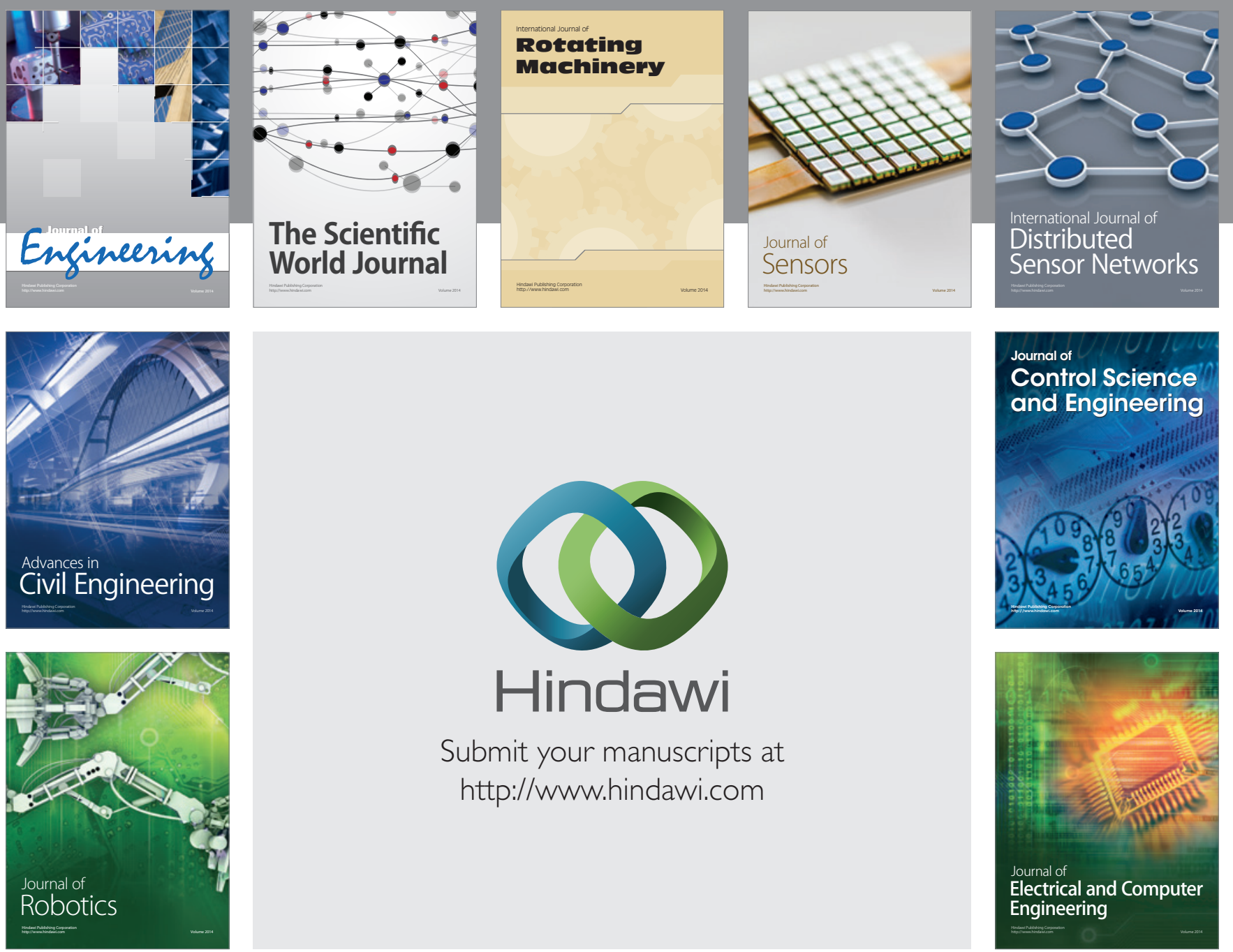

Submit your manuscripts at

http://www.hindawi.com
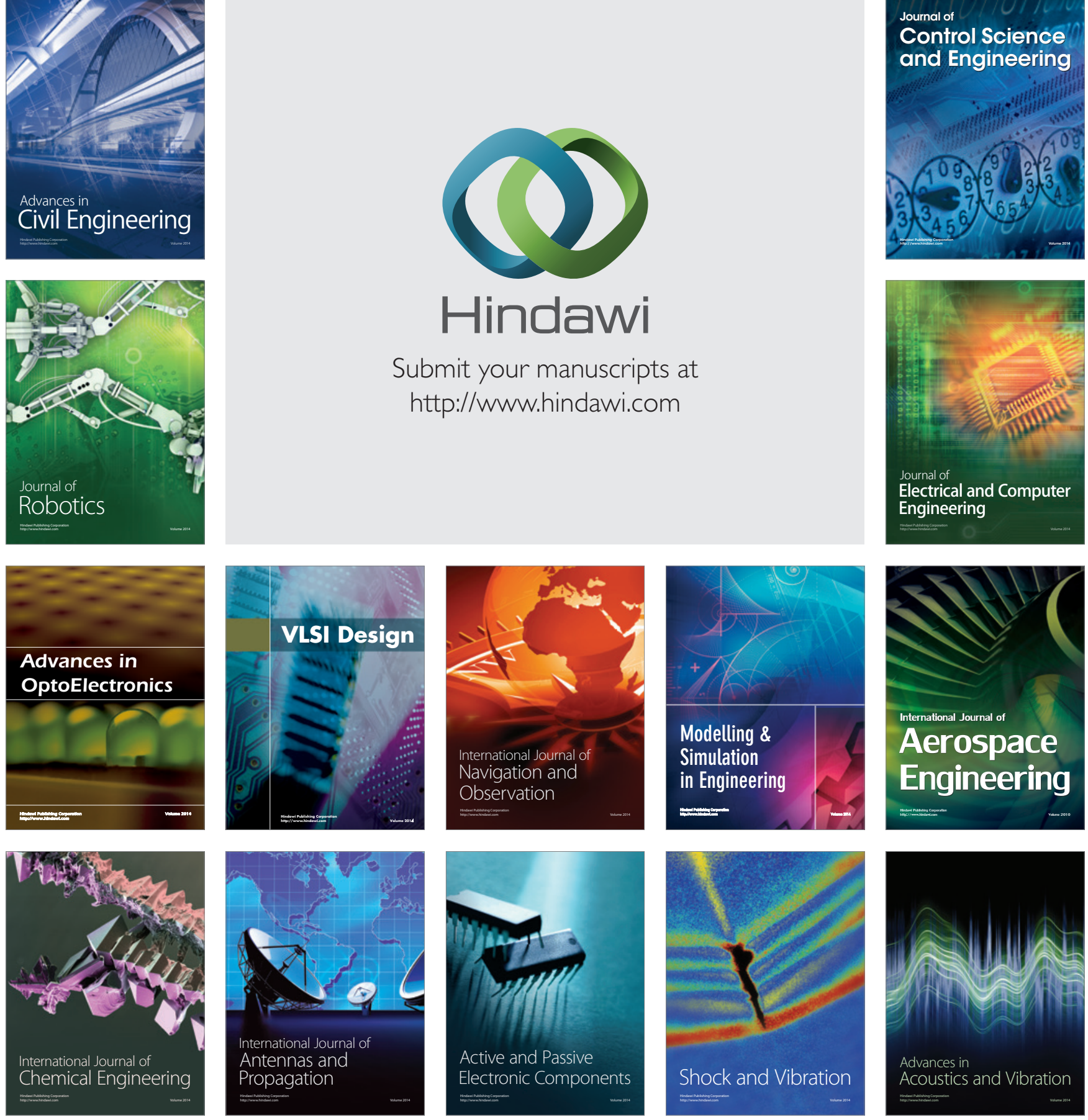